\title{
Dynamics Modeling of the Arc Spring for Powertrain NVH Prediction
}

\author{
Yoshihiro Yamakaji ${ }^{1}$ \\ ${ }^{1}$ Exedy Corporation, Japan, y-yamakaji i@exedy $\cdot c o m$
}

\begin{abstract}
The key value of Model Based Development is to realize capability of quick performance design and simulation at the early phase of development. In this paper, we modeled an arc spring type torsional damper, which has an impact on the torsional vibration characteristics of powertrain in a vehicle. To predict non-linearity of the arc springs, we took a discrete modeling approach using MODELICA and compared its simulation results with the physical test results. We also developed a user-friendly interface with FMIE (Modelon FMI Add-in for Excel) so that a non-expert of physical modeling can run performance design easily and precisely on their own.
\end{abstract}

Keywords: Powertrain, Arc Spring, Modelica, FMI, Nonlinear Vibration

\section{Introduction}

These days, as a car gets more complex than ever, Automotive Original Equipment Manufacturer (OEM, hereafter) are asking Parts Suppliers to deliver performance proposals at the very early phase of development. To do that, it is imperative for Parts Suppliers such as EXEDY to realize performance design with accurate and quick performance prediction.

Recently, Model Based Development (MBD, hereafter) has widely been spread out in Automotive Industry. MBD is an approach to model and simulate systems behaviors taking multiple physical domains into account even before starting the detailed design. To meet the OEM requirements described above, we applied MBD to the development of torsional damper products. The problem here is how to model the arc spring type torsional damper with a high non-linear characteristic. Therefore, we focus on modeling and validation of the arc spring component in a torsional damper.

In EXEDY, we chose some MBD tools capable of handling physical modeling, such as Dymola, for Prediction and Validation phases in V-process shown in Fig. 1. To accelerate its deployment, it is important to build simulation models for better performance and accuracy, and to establish workflow to utilize MBD tools efficiently.
The paper comprises following chapters. First, we explain the basic structure of the torsional damper. Second, we focus on the arc spring and illustrate its physical models. Third, we show Modelica implementation and the comparison with the physical tests. Then we present our interface program on top of Microsoft EXCEL by using FMIE which enables a non-expert of physical modeling to work on the performance design of arc springs based on Modelica.

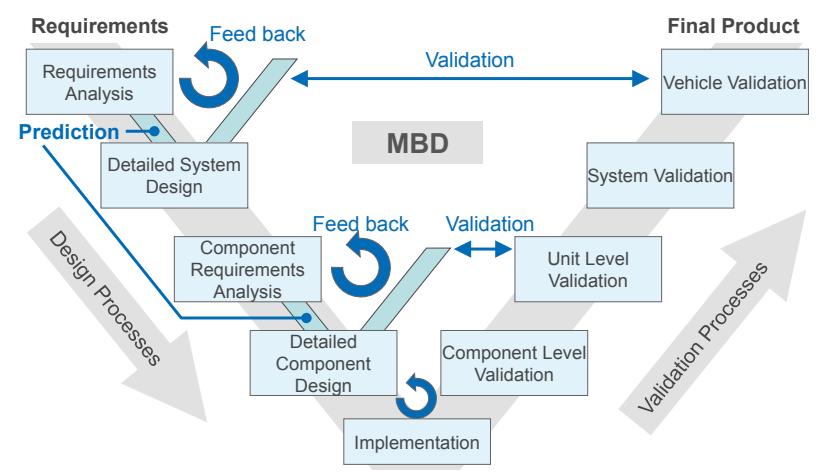

Figure 1. Model-Based-Development Process

\section{Description of Target Systems}

\subsection{Functions of Launch Devices}

Launch devices of a car must provide following four functionalities.

1. Transfer and cut off power

2. Smooth connection

3. Noise-proof and vibration-proof

4. Fuse of drivetrain

In this paper we focus on a torsional damper which plays a key role for the functionality 3 above, noiseproof and vibration-proof.

Torsional damper mitigates the torque fluctuation from a motor such as ICE (Internal Combustion Engine) and delivers only smoothed driving torque to downstream transmission (T/M, hereafter) (Fig. 2). It reduces the torsional vibration of drivetrain which leads to the elimination of gear noise and booming noise. Adoption of recent advanced environmental technology (such as fewer cylinders or turbo chargers) causes more torque fluctuation, which requires the torsional damper to be more effective. 


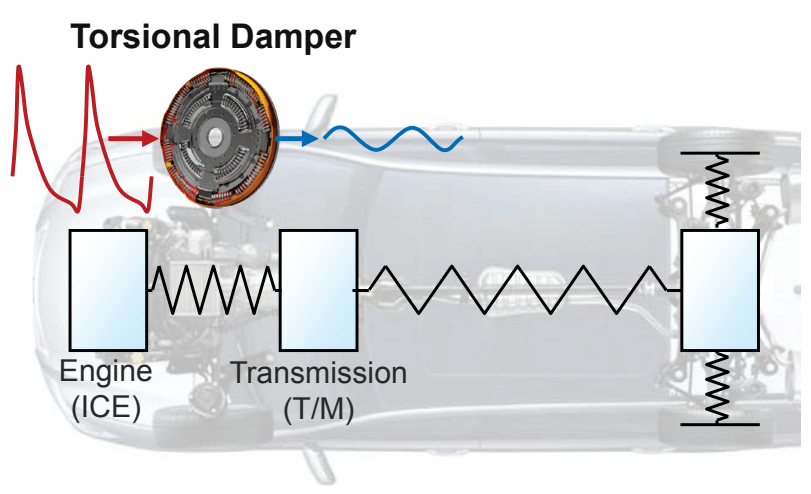

Figure 2. Function of Torsional Damper

\subsection{Torsional Damper}

Torsional dampers typically consist of multiple straight springs or arc springs. When torque input comes in from ICE side, the springs absorb and release torques repeatedly. Through this mechanism, torque fluctuation is rectified to reduce the drivetrain torsional vibration after $\mathrm{T} / \mathrm{M}$.

To optimize drivetrain torsional vibration, it is an effective means to optimize the eigenvalues of torsional vibration of drivetrain. Those eigenvalues are dominated by torsional stiffness of torsional dampers. By setting eigenvalues lower than the driving range (for instance, for Automatic Transmission, under the lock-up lower-limit rotation), silence characteristics during driving is assured (Fig. 3).

However, reducing torsional damper stiffness leads to a constraint on space, because torsional angle range has to be set wider. Also, there is a trade-off by excessive low stiffness, that is, for instance, lowfrequency vibration on the vehicle. Therefore, it is necessary to determine design specifications optimally to satisfy all target performances from OEM.

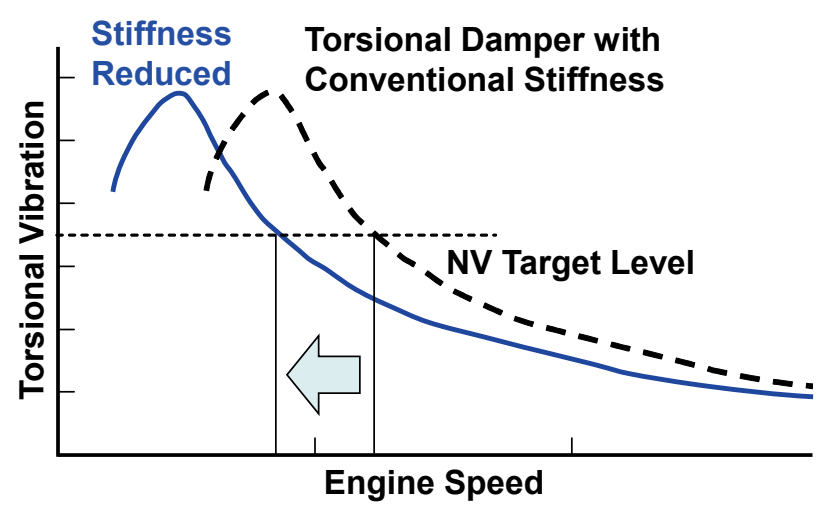

Figure 3. Powertrain Torsional Vibration Mitigation by Torsional Damper Stiffness Reduction

\subsection{Characteristics of Arc Springs}

Arc springs, which are included in the torsional damper, have non-linear damping characteristics. Fig. 4 shows the torque fluctuation against relative torsional angles when we apply certain amplitude of torque to the arc spring. We see by those figures that the hysteresis curve resembles a leaf, which means equivalent stiffness and damping coefficients dynamically change depending on rotational speed or input torque amplitude. It is necessary to develop a highly accurate and predictive model to virtually reproduce such hysteresis curves.

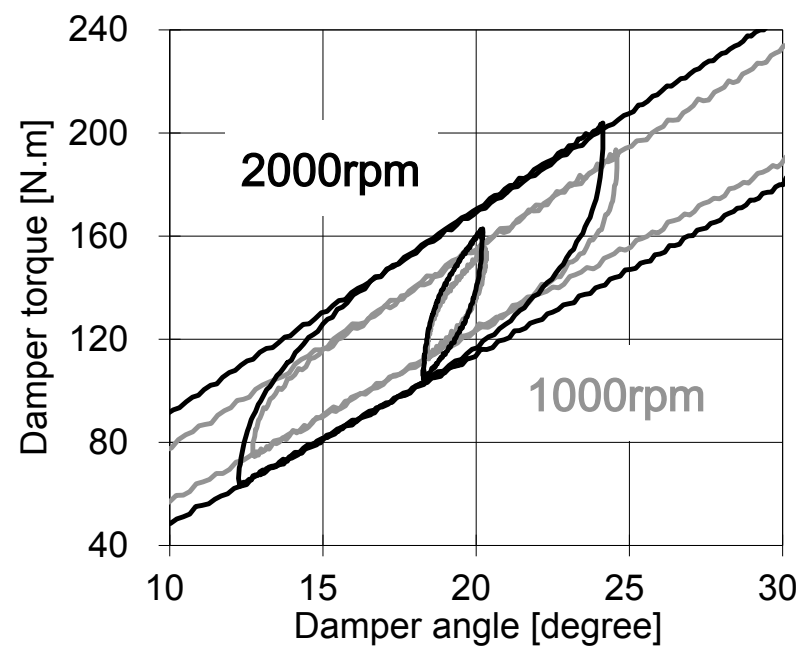

Figure 4. Dynamic Characteristics of Arc Springs

Torsional torque over the relative angle at 1000rpm and 2000 rpm on Component Level Test(Experimental data)

\section{Modeling Arc Springs}

To understand the measured characteristics (hysteresis curve) of arc springs, we use $n_{E L M}$-number of linear spring elements $k_{n}$ and mass elements $m_{n}$ to discretize a continuous spring element (Fig. 5). Here, $n$ is the element number counted from the input torque side, $k_{n}$ is the discretized stiffness which is the overall spring ratio multiplied by $n_{E L M}, m_{n}$ is the discretized spring mass which is the overall mass divided by $n_{E L M}$.

We also define that an arc spring is stored in a cylindrical container in which the inner diameter is the same size as the arc of the spring's outer diameter. One end of the arc spring is connected to the input element, and the end of the other side is coupled to the cylindrical container so as not to rotate relatively. The cylindrical container is connected to the output element. The angle limitation is not included in this modeling.

Considering that a torsional damper is rotating at some speed, centrifugal force is applied onto the mass element which is consequently pressed against the cylindrical container. If a mass element rotates relatively to the cylindrical container because of the input torque to the arc spring, friction torque $T_{F n}$ would be caused not only by the centrifugal load but also by the reaction force of the arc spring. 


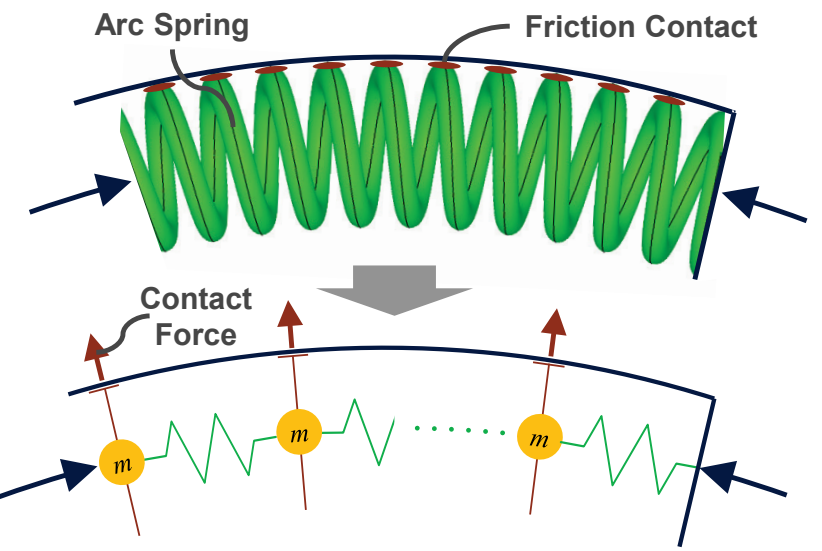

Figure 5. Discretized Model for Arc Springs

\subsection{Mechanism Identification}

Figure 6 shows the relation of torque and the relative torsional angle between input and output elements and the estimated mechanism, when torque $T_{i n}$ is added to a discretized model of a torsional damper. From the figures, we define the relation between $T_{\text {in }}$ and output torque $T_{\text {out }}$ in several steps.

1. $T_{\text {in }}<T_{F 1}$ :

$T_{\text {in }}$ is transmitted to the output side by the friction torque $T_{F 1}$ from a mass element $m_{1}$. Torque is transmitted in a state where mass element $m_{1}$ and the output element are coupled, hence $T_{\text {out }}=T_{\text {in }}$.

2. $T_{\text {in }}>T_{F 1} A N D T_{k 1}<T_{F 2}$

$T_{i n}$ is transmitted by $T_{F 1}$ and friction torque $T_{F 2}$ of $m_{2}$. Here $m_{1}$ slips relative to the output element, but $m_{2}$ is coupled to the output element. Input torque on $m_{2}$ is torsional torque on the spring element $k_{1} ; T_{k 1}=T_{i n}-T_{F 1}$;. At this step, the torsional stiffness is composed of $k_{1}$.

3. $T_{i n}>T_{F 1}+T_{F 2}$ AND $T_{k 2}<T_{F 3}$

$m_{1}$ and $m_{2}$ slip relatively to the output element, then $k_{1}$ and $k_{2}$ work. At this step, the torsional stiffness is composed of the direct stiffness by $k_{1}$ and $k_{2}$, so the slope represented the relation between torque and angle (= torsional torque) becomes smaller than condition 2 .

4. For other elements, elements gradually move relative to the output elements by the relation between input torque and friction torque. When input torque becomes larger than all mass elements' friction torque, all spring elements will move. Every time the relative torsional direction is reversed caused by the fluctuation of the input torque, the motion is reset and restarts from the step 1.

By the mechanism identified above, we consider that the measured characteristics (a leaf shape hysteresis curve) appear.

Now we define the force equation based on the identified mechanism. The friction torque of one mass element is defined as the equation below:

$$
T_{F n}=\mu_{D} \cdot r_{F} \cdot F_{R n}, \quad n=1, \ldots, n_{E L M}
$$

Here, $T_{F n}$ is friction torque associated with each mass element, $\mu_{D}$ is a dynamic friction coefficient, $r_{F}$ is a friction radius, $F_{R n}$ is a pressing force to the friction surface.

The pressing force to the friction surface is distributed as shown in Fig. 7, and defined per the equations below:

$$
F_{R n}=F_{C n}+F_{S N n}+F_{S N(n-1)}
$$

Where:

$$
\begin{aligned}
n & =2, \ldots, n_{E L M} \\
F_{C n} & =m_{n} \cdot r_{A} \cdot \omega^{2} \\
F_{S N n} & =F_{S n} \cdot \sin \varphi_{S n} \\
F_{S n} & =k_{S n} \cdot r_{A} \cdot \sin \theta_{S n}
\end{aligned}
$$

Also:

$$
F_{S N 1}=\frac{T_{i n}}{r_{A}} \sin 0=0
$$
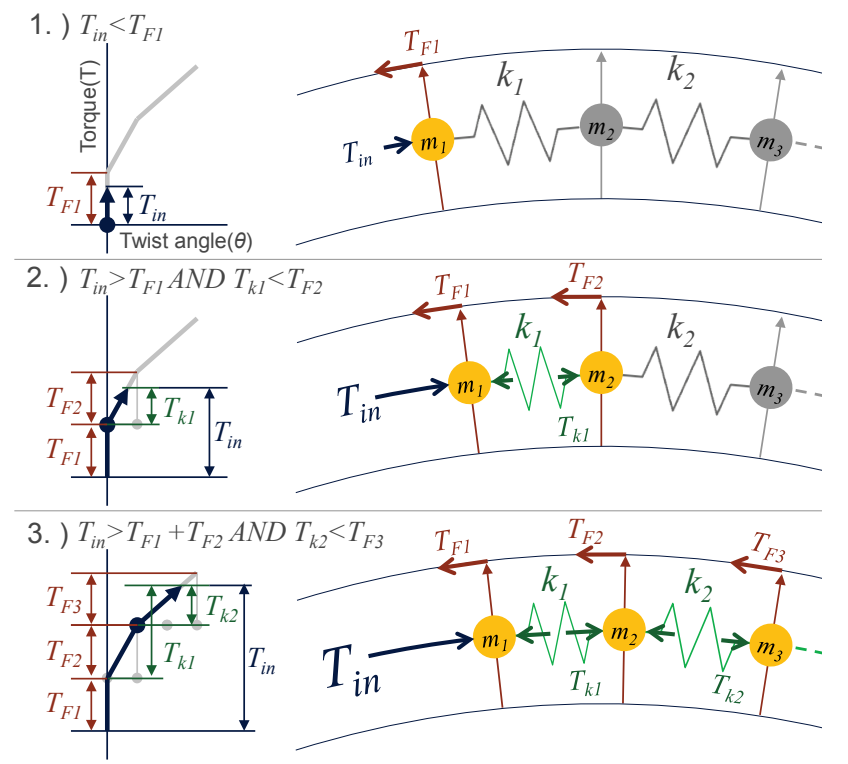

Figure 6. Momentary Behavior of Arc Springs

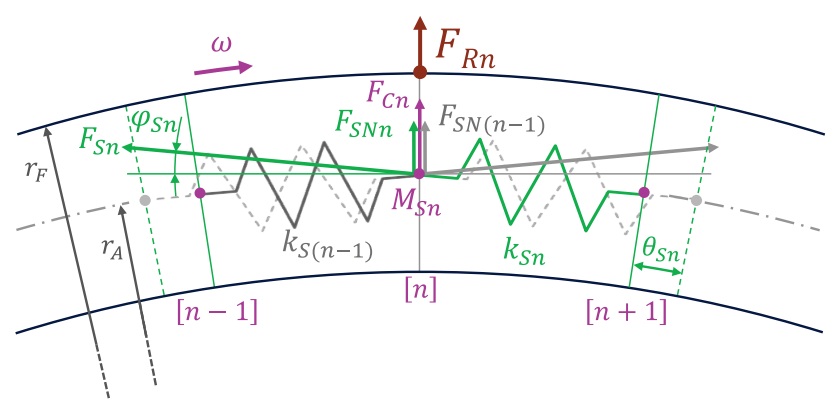

Figure 7. Modeling of Pressing Force on Mass Element 


\subsection{Study of Discretization Level}

The arc spring is wound up dozens of times. Therefore it is important to determine the number of elements for discretization. If we take the actual number of turns for discretization, the simulation model will have several degrees of freedom only with an arc spring. This may lead to high accuracy but slower calculation speed. When the discretization number is too small, the problem comes into calculation accuracy and vice versa (because the detail behavior shown in 3.1 is not reproduced).

So we investigate how much $T_{F}$ will be impacted depending on the change of $n_{E L M}$, with representative torsional damper design variables to equation (1) and (2). Fig. 8 shows prediction accuracy versus numbers of elements; here, when $n_{E L M}$ is set as an actual winding number, prediction accuracy of $T_{F}$ is defined as $100 \%$. By the figure, prediction accuracy is expected more than $98 \%$, when the number of elements is more than 6 .

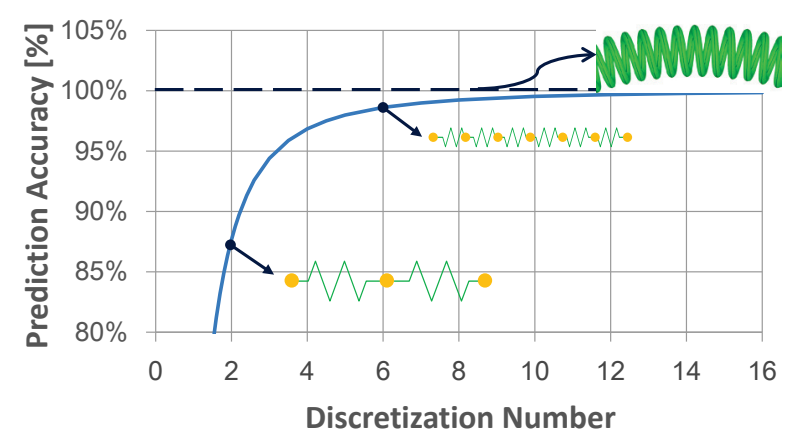

Figure 8. Relation between prediction accuracy and discretization level

When $\boldsymbol{n}_{E L M}$ is set as an actual winding number, prediction accuracy of $\boldsymbol{T}_{\boldsymbol{F}}$ is defined as $100 \%$.

\section{Modeling \& Verification}

Modelica was chosen to implement the considered mechanism. The reason for this is that compared to other physical modeling tools, we get the following benefits:

- Straightforward description with equations

- Simple mixing of equations and physical models

- Reuse and extension of models due to expressiveness of the source code

- In-house built package can be integrated

\subsection{Modeling Arc Spring Components}

First, the spring and mass elements modeled using the SpringDamper and Inertia component are already available in the Mechanics.Rotational package included in the Modelica Standard Library (MSL). Internal variables are then bound to output signals so they can be used in the friction torque equations.
Next, the observer component computing the friction torque is created. Equation (1) is rewritten in Modelica code. The spring element information to be used as variables are retrieved from the MSL component outputs. Once computed, the friction torque is also exposed as an output.

When friction torque occurs, internal friction torque component is used. It generates the friction torque according to the output value computed by the observer component.

The arc spring component is based on these subcomponents. In order to validate the estimation accuracy depending on the variation of $n_{E L M}$, we create several arc spring modules based on different values for $n_{E L M}$.

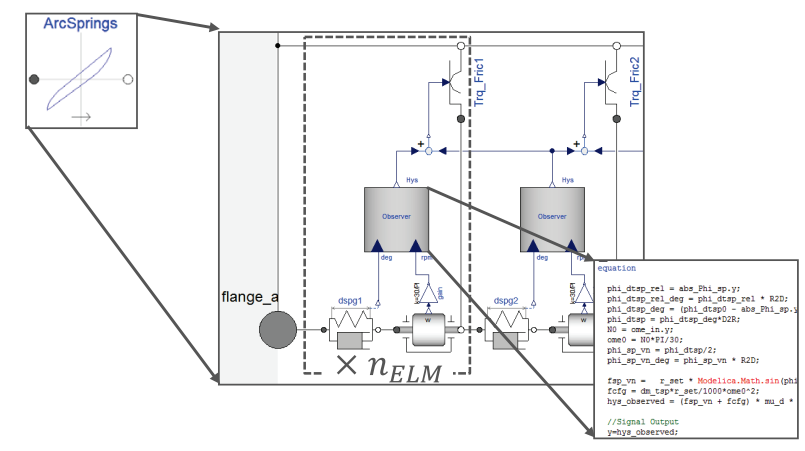

Figure 9. Arc spring component implementation in Modelica

The component is made of 4 subcomponents (SpringDamper, Inertia extended with extra outputs, Friction Torque Observer with the mechanism equations, and FrictionTorque).

\subsection{Component Level Verification}

In order to confirm the arc spring module correctness and precision, simulations reproducing unit test equivalent to Fig. 4 are run and we compared the obtained results.

On Fig. 10 and Fig. 11, results from a simulation run with $n_{E L M}=8$ are compared with experimental measurements from Fig. 4. We can observe that the data is mostly matching, and that the input torque oscillations are varying according to a non-linear pattern.

Comparisons for $n_{E L M}=1 \ldots 10$ are shown on Fig. 12. When $n_{E L M}$ is below 6 , the non-linearity is not well-preserved and the precision loss observed on Fig. 8 is confirmed.

When comparing the number of generated equations and the overall computation time, it appears that time grows quadratically with the number. To keep reasonable simulation times it is important to have the discretization that would give us a good balance between accuracy and computation cost.

Confronting those results, we can validate the model we built. 


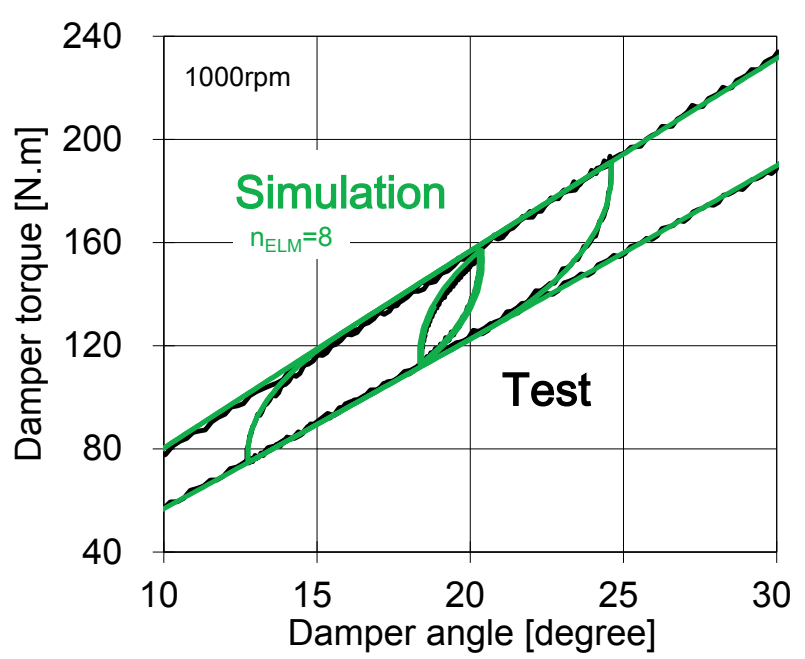

Figure 10. Arc spring model validation (1)

Simulated vs. experimental data for $n_{E L M}=8$ at $1000 \mathrm{rpm}$

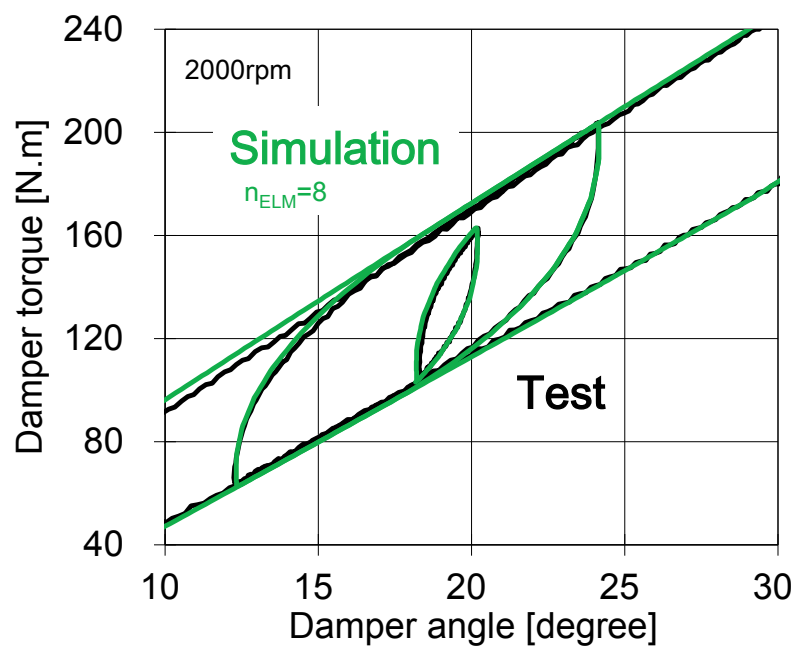

Figure 11. Arc spring model validation(2)

Simulated $v$ s. experimental data for $n_{E L M}=8$ at $2000 \mathrm{rpm}$

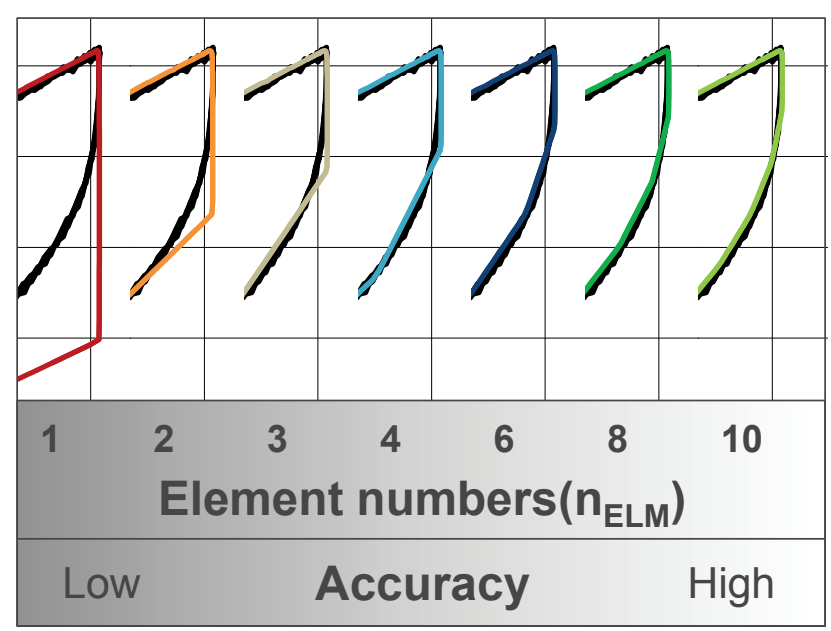

(a) Simulated torsional characteristic (colored lines) compared by measurement data (black line)

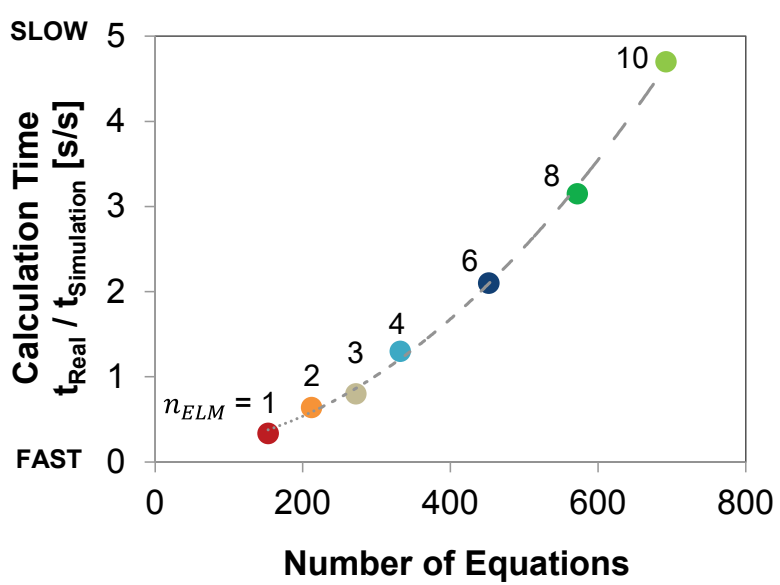

(b) Relation between calculation time and number of equations

Figure 12. Comparison with each discretized level

\section{Physical model deployment}

To make highly accurate physical models accessible to a larger number of engineers, a consistent and easy-touse interface is required. If the operation varies depending on physical modeling tools, it is ineffective in total because all the work must be done by physical modeling experts.

The capability of having a model run in black-box is also essential. Physical modeling tool seems easy to users and they might carelessly connect components in an unintended way. Behaviors and errors which are not intended by model developers must be avoided at all cost.

By using the Functional Mock-up Interface (referred as FMI from below) as a standard to connect models, we established a process which enables everyone to conduct performance prediction by physical models. Users may only interact with a generic Microsoft Excel interface using the Modelon FMI Add-in for Excel (FMIE). We choose Co-Simulation to export the arc spring model because the binary export license of Dymola is not needed.

FMIE can read FMI 1.0 models exported from MODELICA-based tools such as Dymola, can choose input or output variables, set up scenarios to be run, and execute the simulation, and return the results. However, going through these steps every time is inefficient. So we developed a VBA macro (Fig. 14) to partially automate the process. Once we provide design variables and simulation parameters and press a button, all the relevant simulations are performed and result graphics generated (Fig. 15). With this, anyone can simply run quality simulations only interacting with Excel, without any physical modeling tool even running in the background. 


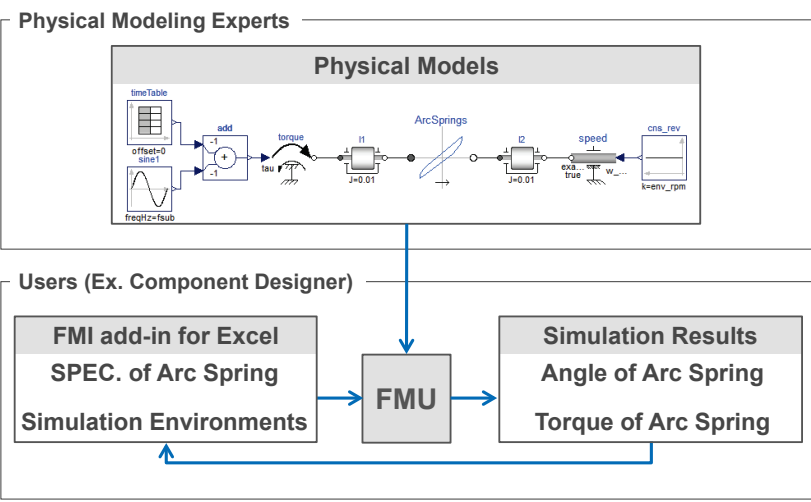

Figure 13. Fast Simulation Method using FMIE

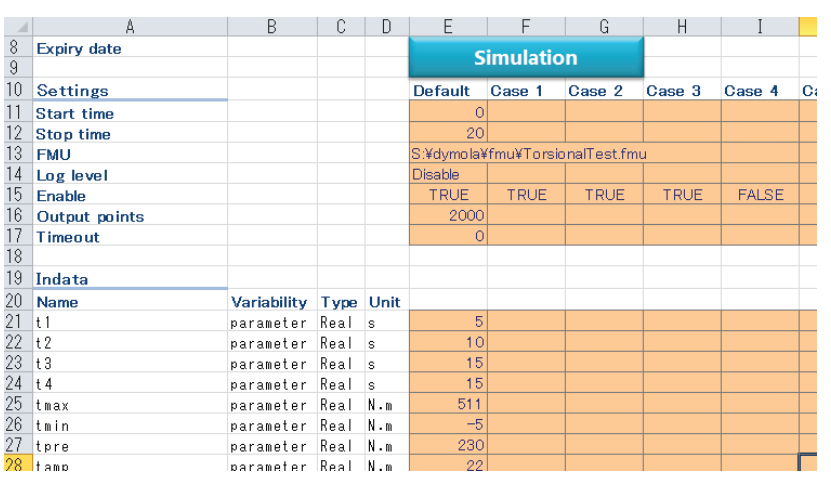

Figure 14. Screenshot of FMIE embedding VBA

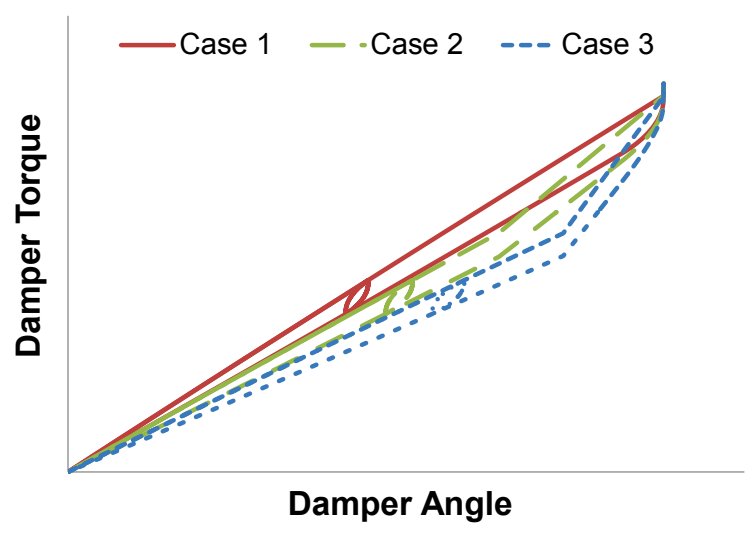

Figure 15. Example of prediction results using FMIE

\section{Conclusion}

- We developed a discretized model with MODELICA which represents non-linearity of arc springs

- We verified the model accuracy by comparing the dynamic simulation results with measured results of physical tests at component level

- We developed an intuitive interface to design arc spring performance easily and accurately

Those outcomes enable non-experts of physical modeling to run performance design easily with high accuracy. It will lead to the cost reduction of human resources and speed up product development.

\section{Acknowledgements}

We gratefully acknowledge the support of Mr. Okabe from Dassault Systèmes and Mr. Gao from Modelon to complete this paper with their technical advices and translation.

\section{References}

Dr.-Ing. Albert Albers. Advanced Development of Dual Mass Flywheel, LuK Symposium book, 1994.

Shinji Hounoki, Katsuyuki Kobayashi, Mitsuhiro Umeyama, Toshihiro Otake. Study of the Two-Mass Flywheel with the Torsional Damper. Society of Automotive Engineers of Japan (JSAE) Proceedings, 902138-1:157-160 , 1990.

Ulf Schaper, Oliver Sawodny, Tobias Mahl and Uti Blessing. Modeling and torque estimation of an automotive Dual Mass Flywheel. 2009 American Control Conference, 9781-4244-4524-0/09 (WeB16.6 1207-1212), Hyatt Regency Riverfront, St. Louis, MO, USA, June 10-12, 2009.

Yasuo Shimizu, Nobutaka Tsujiuchi, Akihito Ito, Satoshi Yamamoto. Reduction of Low Frequency vibration at Acceleration/Deceleration by Optimization of Flywheel Damper for Passenger Car. Society of Automotive Engineers of Japan (JSAE), 19-15A:454-459, 2015.

Yamakaji Yoshihiro. Approaches to quick prediction for Powertrain NVH. 9th International CTI Symposium North America, Novi, USA, 2015-05-20/21 